Faculty of Mathematical Sciences

\section{University of Twente}

University for Technical and Social Sciences
P.O. Box 217

7500 AE Enschede

The Netherlands

Phone: +31-53-4893400

Fax: +31-53-4893114

Email: memo@math.utwente.nl

Memorandum No. 1491

On factors of 4-connected claw-free graphs

H.J. Broersma, M. Kriesell and Z. RyjáčeK ${ }^{1}$

JULY 1999

ISSN 0169-2690

\footnotetext{
${ }^{1}$ Department of Mathematics, University of West Bohemia, Univerzitní 22, 30614 Plzeň, Czech Republic
} 


\title{
On factors of 4-connected claw-free graphs
}

\author{
H. J. Broersma \\ M. KRIESELL \\ Faculty of Mathematical Sciences \\ University of Twente \\ P.O. Box 217, 7500 AE Enschede, The Netherlands \\ Z. RYJÁČEK* \\ Department of Mathematics \\ University of West Bohemia \\ Univerzitní 22, 30614 Plzeň, Czech Republic
}

July 15, 1999

\begin{abstract}
We consider the existence of several different kinds of factors in 4-connected claw-free graphs. This is motivated by the following two conjectures which are in fact equivalent by a recent result of the third author. Conjecture 1 (Thomassen): Every 4-connected line graph is hamiltonian, i.e. has a connected 2-factor. Conjecture 2 (Matthews and Sumner): Every 4-connected claw-free graph is hamiltonian. We first show that Conjecture 2 is true within the class of hourglass-free graphs, i.e. graphs that do not contain an induced subgraph isomorphic to two triangles meeting in exactly one vertex. Next we show that a weaker form of Conjecture 2 is true, in which the conclusion is replaced by the conclusion that there exists a connected spanning subgraph in which each vertex has degree two or four. Finally we show that Conjecture 1 and 2 are equivalent to seemingly weaker conjectures in which the conclusion is replaced by the conclusion that there exists a spanning subgraph consisting of a bounded number of paths.
\end{abstract}

Keywords: claw-free graph, line graph, Hamilton cycle, Hamilton path, factor

AMS Subject Classifications (1991): 05C45, 05C38

\section{Introduction}

We use [1] for terminology and notation not defined here.

Most of the results in this paper are motivated by the following two conjectures due to Thomassen [13] and Matthews And Sumner [10], respectively.

\footnotetext{
${ }^{*}$ Research supported by grant GA ČR No. 201/97/0407
} 


\section{Conjecture 1}

Every 4-connected line graph is hamiltonian.

\section{Conjecture 2}

Every 4-connected claw-free graph is hamiltonian.

A recent result on closures due to the third author [11] (Theorem 3 below) implies that Conjecture 1 and Conjecture 2 are equivalent.

We first introduce some terminology and notation. All multigraphs considered in the sequel are finite, undirected, and loopless. We use the term graph for a multigraph $G=(V, E)$ in order to indicate that $G$ is simple, i.e. there is at most one edge joining two vertices. As usual, $V(G)$ or $V$ denotes the vertex set and $E(G)$ or $E$ the edge set of a multigraph $G$. Let $A, B \subseteq V$ and $a, b \in V$. With $[A, B]_{G}$ we denote the set of edges between vertices of $A$ and $B$ in $G$, and we let $[a, b]_{G}:=[\{a\},\{b\}]_{G}$. If $[a, b]_{G}=\{e\}$ for some $e \in E$, then we also use $a b$ or $[a, b]_{G}$ for $e$.

The submultigraph $G[A]$ induced by the set $A \subseteq V(G)$ is defined by $G[A]:=\left(A,[A, A]_{G}\right)$, and the degree of some vertex $a \in V$ is denoted by $d_{G}(a):=\left|[\{a\}, V \backslash\{a\}]_{G}\right|$. Let $N_{G}(A):=$ $\left\{c \in V \backslash A \mid[A,\{c\}]_{G} \neq \emptyset\right\}$, and let $N_{G}(a):=N_{G}(\{a\})$. Clearly, $d_{G}(a)=\left|N_{G}(a)\right|$ provided that $G$ is a graph. The submultigraph $G\left[N_{G}(a)\right]$ is called the neighborhood of $a$ in $G$. By $d_{G}(a, b)$ we denote the distance of $a, b$ in $G$, i.e. the length of a shortest path between $a$ and $b$ in $G$. If $a, b$ are not in the same component of $G$, we simply set $d_{G}(a, b):=\infty$.

A claw in the multigraph $G$ is a set of four distinct vertices $a, b, c, y$ such that $a, b, c$ are independent in $G$, i.e. pairwise nonadjacent in $G$, and $a, b, c \in N_{G}(y) . G$ is called claw-free if there exists no claw in $G$. Clearly, a multigraph is claw-free if it contains no induced subgraph isomorphic to $K_{1,3}$, but the converse is guaranteed only in graphs.

A spanning submultigraph $H$ of $G$ is called a factor of $G$, and a 2-factor (of $G$ ) if all vertices of $H$ have degree 2 in $H$. Hence a Hamilton cycle is a connected 2-factor. A circuit $C$ of $G$ is a closed trail (possibly consisting of a single vertex), and it is said to be (edge) dominating if every edge of $G$ is incident with some vertex of $C$. If, moreover, $V(G)=V(C)$ holds then $C$ is a spanning circuit.

The local completion of a graph $G$ at a vertex $v$ is the operation of joining all pairs of nonadjacent vertices in $N_{G}(v)$, i.e. replacing the neighborhood of $v$ by the complete graph on $N_{G}(v)$.

In [11] the following has been proved.

\section{Theorem 3}

Let $G$ be a claw-free graph, $v$ a vertex of $G$ whose neighborhood is connected, and $G^{\prime}$ the graph obtained from $G$ by local completion at $v$. Then

(i) $G^{\prime}$ is claw-free, and

(ii) for every cycle $C^{\prime}$ of $G^{\prime}$ there exists a cycle $C$ of $G$ such that $V\left(C^{\prime}\right) \subseteq V(C)$. 
For a claw-free graph $G$, we define the closure $\operatorname{cl}(G)$ of $G$ as the graph obtained from $G$ by iteratively performing local completions at vertices with connected neighborhoods until no more edges can be added. As shown in [11], $\operatorname{cl}(G)$ is uniquely determined by $G$, and $\operatorname{cl}(G)$ is the line graph of a triangle-free graph. Moreover, in [11] it is shown that Theorem 3 has the following consequences. Let $c(G)$ denote the circumference of $G$, i.e. the length of a longest cycle of $G$.

\section{Theorem 4}

Let $G$ be a claw-free graph. Then

(i) $c(c l(G))=c(G)$.

(ii) If $\mathrm{cl}(G)$ is complete and $|V(G)| \geq 3$, then $G$ is hamiltonian.

(iii) Every nonhamiltonian claw-free graph is a factor of a nonhamiltonian line graph.

Theorem 4(iii) together with a result of ZHAN [15] and, independently, JACKSON [5] implies that every 7 -connected claw-free graph is hamiltonian. Moreover it yields the mentioned equivalence of Conjecture 1 and Conjecture 2.

In the sequel we prove several results concerning the existence of certain factors in 4connected claw-free graphs or multigraphs.

In the next section we give a short proof of Conjecture 2 within the subclass of hourglassfree graphs, i.e. graphs that do not contain an induced subgraph isomorphic to the hourglass, a graph consisting of two triangles meeting in exactly one vertex. This result also follows from a recent result due to the second author [7].

In Section 3 we prove the validity of a weaker form of Conjecture 2 in which we replace the conclusion by the conclusion that there exists a connected factor in which each vertex has degree 2 or 4 .

Finally, in Section 4 we show that Conjecture 1 and 2 are equivalent to seemingly weaker conjectures in which we replace the conclusion by the conclusion that there exists a factor consisting of a bounded number of paths.

\section{Hourglass-free graphs}

Our aim in this section is to prove that all 4-connected claw-free hourglass-free graphs are hamiltonian. For this purpose we need the fact that all 4-connected inflations are hamiltonian.

We start this section by introducing some additional terminology. A multigraph $G$ is called essentially $k$-edge connected if it is connected and if every edge cut $E^{\prime}$ of $G$ such that $G-E^{\prime}$ has at least two components containing an edge, has at least $k$ edges. It is well-known and easy to check that a line graph $L(G)$ of a multigraph $G$ is $k$-connected if and only if $G$ is essentially $k$ edge connected. The inflation $I(G)$ of a graph $G$ is the graph obtained from $G$ by replacing all vertices $v_{1}, v_{2}, \ldots, v_{n}$ of $G$ by disjoint complete graphs on $d\left(v_{i}\right)$ vertices $v_{i, 1}, v_{i, 2}, \ldots v_{i, d\left(v_{i}\right)}$, and all edges $v_{i} v_{j}$ by disjoint edges $v_{i, p} v_{j, q}\left(i, j \in\{1, \ldots, n\} ; p \in\left\{1, \ldots, d\left(v_{i}\right)\right\} ; q \in\left\{1, \ldots, d\left(v_{j}\right)\right\}\right)$. 
Alternatively, as shown in [10, Lemma 2], $I(G)$ is the line graph of the subdivision graph $S(G)$, i.e. the graph obtained from $G$ by subdividing each edge of $G$ once. We use the term inflation for a graph that is isomorphic to the inflation of some graph. It is obvious that inflations are claw-free and hourglass-free.

The following result has been observed by several graph theorists, but we have not found it in literature (therefore, we include its proof).

\section{Lemma 5}

Every 4-connected inflation is hamiltonian.

Proof Let $G$ be a 4-connected inflation. Then $G=L(S(H))$ for some essentially 4-edge connected subdivision $S(H)$ of a 4-edge connected graph $H$. As shown in [13], using the result of Kundu [8] that $H$ has two edge-disjoint spanning trees, it is easy to show that $H$ contains a spanning circuit, hence $S(H)$ contains a dominating circuit. By a result of HARARY AND NASH-Williams [3] this implies $G=L(S(H))$ is hamiltonian.

The connectivity bound in Lemma 5 cannot be decreased, since there are nonhamiltonian 3 -connected inflations, e.g. the inflation of the Petersen graph. These graphs also show that the connectivity bound in the next result is best possible.

\section{Theorem 6}

Every 4-connected claw-free hourglass-free graph is hamiltonian.

Proof Let $G$ be a 4-connected claw-free hourglass-free graph. Then by a result in $[2] \operatorname{cl}(G)$ is also claw-free and hourglass-free. Hence by Theorem 4 we can assume that $G=\operatorname{cl}(G)$. This implies that the neighborhood of each vertex of $G$ induces either a complete graph or a disjoint union of two complete graphs. Since $G$ is hourglass-free, in the latter case one of the complete graphs is a $K_{1}$. Hence $G$ contains two types of edges, namely edges that are contained in a complete subgraph on more than 2 vertices, and edges that are contained in a $K_{2}$ only. Moreover, all maximal complete subgraphs on more than two vertices contain two types of vertices, namely vertices with a complete neighborhood (contained in the subgraph) which are called simplicial vertices, and vertices with precisely one neighbor outside the subgraph. It is not difficult to check that the graph $G^{\prime}$ obtained from $G$ by deleting all simplicial vertices is a 4-connected inflation. Hence $G^{\prime}$ is hamiltonian by Lemma 5. Clearly, a Hamilton cycle in $G^{\prime}$ contains at least one edge of each maximal complete subgraph on more than 2 vertices, and all the maximal complete subgraphs of $G$ containing simplicial vertices correspond to such subgraphs. Hence a Hamilton cycle of $G^{\prime}$ can easily be extended to a Hamilton cycle in G.

\section{Connected factors with degree restrictions}

By Theorem 3.1 in [6], every connected claw-free graph has a 2-walk, i.e. a (closed) walk which passes every vertex at most twice. Clearly, the edges of a 2-walk induce a connected factor of maximum degree at most 4 . 
The aim of this section is to prove that every 4-connected claw-free graph contains a connected factor with vertices of degree 2 or 4 . We start with a series of lemmas on congruent factors of multigraphs, i.e. factors of a multigraph $G$ which have the same parity of degrees at every vertex. Lemma 7 will allow us to apply the closure introduced in Section 1 later on. (Note that $\operatorname{cl}(G)$ can be constructed from $G$ by iteratively adding the missing edge in a subgraph $K_{4}-e$.)

\section{Lemma 7}

Let $F$ be a connected factor of a multigraph $G$ and let $e$ be an edge contained in some complete subgraph $K_{4}$ of $G$. Then $G-e$ has a connected factor $F^{\prime}$ such that $d_{F^{\prime}}(x) \equiv d_{F}(x) \bmod 2$ for all $x \in V(G)$.

Proof For two multigraphs $G_{1}, G_{2}$ we define $G_{1} \cup G_{2}:=\left(V\left(G_{1}\right) \cup V\left(G_{2}\right), E\left(G_{1}\right) \cup E\left(G_{2}\right)\right)$, $G_{1} \cap G_{2}:=\left(V\left(G_{1}\right) \cap V\left(G_{2}\right), E\left(G_{1}\right) \cap E\left(G_{2}\right)\right)$, and $G_{1} \Delta G_{2}:=\left(G_{1} \cup G_{2}\right)-E\left(G_{1} \cap G_{2}\right) .\left(G_{1} \Delta G_{2}\right.$ is the symmetric difference of $G_{1}$ and $G_{2}$.)

Let $w, x, y, z$ be the vertices of the subgraph $H \cong K_{4}$ which contains $e$, say $e \in[w, x]$. The conclusion of the lemma is obviously true if $e \notin E(F)$. So we may assume $e \in E(F)$. We define the following four $w, x$-subpaths of $H: Q:=w, y, x, R:=w, z, x, S:=w, y, z, x$, and $T:=w, z, y, x$. It is easy to see that if $F^{\prime}$ is the symmetric difference of $F-e$ and any of these paths, then $d_{F^{\prime}}(u) \equiv d_{F}(u) \bmod 2$ holds for all $u \in V(H)$. Hence it suffices to prove that the symmetric difference $F^{\prime}$ of one of these paths and $F-e$ contains a connected spanning subgraph of $H$. We denote $(F-e) \cap H$ by $H^{\prime}$.

If $d_{H^{\prime}}(y)=3$, then $F^{\prime}:=(F-e) \Delta R$ will serve, if $d_{H^{\prime}}(y)=0$ and $d_{H^{\prime}}(z) \neq 0$ then $F^{\prime}:=(F-e) \Delta Q$ will do, and if $d_{H^{\prime}}(y)=d_{H^{\prime}}(z)=0$ then $D^{\prime}:=(F-e) \Delta T$ will. So we may assume that $y$ and, by symmetry, $z$ have degree 1 or 2 in $H^{\prime}$.

Without loss of generality, we may assume that $d_{H^{\prime}}(w) \geq d_{H^{\prime}}(x)$. We distinguish three cases.

Case 1. $d_{H^{\prime}}(w)=2$ and $d_{H^{\prime}}(x) \geq 1$. Without loss of generality, $x$ is adjacent to $y$ in $H^{\prime}$. Since $d_{H^{\prime}}(y) \neq 3$, there is no edge between $y$ and $z$ in $H$. It follows that $F^{\prime}:=(F-e) \Delta S$ is an appropriate factor.

Case 2. $d_{H^{\prime}}(w)=2$ and $d_{H^{\prime}}(x)=0$. If $y$ is adjacent to $z$ in $H^{\prime}$, then $F^{\prime}:=(F-e) \Delta Q$ will do; otherwise $F^{\prime}:=(F-e) \Delta S$ will.

Case 3. $d_{H^{\prime}}(w)=1$. Without loss of generality, $w$ is adjacent to $y$ in $H$. If $x$ is not adjacent to $z$, then $F^{\prime}:=(F-e) \Delta R$ will do; in the other case, $d_{H^{\prime}}(x)=1$ as well, and $F^{\prime}:=(F-e) \Delta T$ contains a connected spanning subgraph of $H^{\prime}$, since it contains all edges of $H^{\prime}$ except possibly an edge beetween $y, z$.

Lemma 8 guarantees the existence of a connected low degree factor in a claw-free multigraph which is congruent to a given one.

\section{Lemma 8}

Let $F$ be a connected factor of a claw-free multigraph $G$. Then there exists a connected factor $F^{\prime}$ of $G$ without vertices of degree exceeding 4 such that $d_{F^{\prime}}(x) \equiv d_{F}(x) \bmod 2$ for all $x \in V(G)$. 
Proof Throughout the proof, we call a connected factor $F^{\prime}$ with $d_{F^{\prime}}(x) \equiv d_{F}(x) \bmod 2$ for all $x \in V(G)$ a good factor. Among all good factors we choose one, say $F^{\prime}$, with a minimum number of edges. We claim that $F^{\prime}$ contains no vertex of degree exceeding 4 .

Suppose to the contrary that $x \in V(G)$ had degree at least 5 in $F^{\prime}$. We distinguish two cases.

Case 1. $F^{\prime}-x$ is connected. First note that there is no pair of distinct edges $e, f \in E\left(F^{\prime}\right)$ between $x$ and some $y \in V(G)$, for otherwise $F^{\prime}-e-f$ would be a good factor, contradicting the choice of $F$. So $\left|N_{F^{\prime}}(x)\right| \geq 5$. Let $e \in[y, z]_{G}$ be an edge in $G\left[N_{F^{\prime}}(x)\right]$. Then $e \in E\left(F^{\prime}\right)$, too, for otherwise $\left(F^{\prime}-[x, y]-[x, z]\right)+e$ would be a good factor, a contradiction. Furthermore, $e$ is a bridge of $F^{\prime}-x$, for otherwise $F^{\prime}-[x, y]-[x, z]-e$ is a good factor, which is absurd again. So every edge in $G\left[N_{F^{\prime}}(x)\right]$ is a bridge of $F^{\prime}-x$, and in particular, $G\left[N_{F^{\prime}}(x)\right]$ contains no cycle. But then $N_{F^{\prime}}(x)$ must contain three independent vertices (since $\left|N_{F^{\prime}}(x)\right| \geq 5$ ), which form a claw together with $x$, a contradiction.

Case 2. $F^{\prime}-x$ is not connected. First note that there is no triple $e, f, h \in E\left(F^{\prime}\right)$ between $x$ and some $y \in V(G)$, for otherwise $F^{\prime}-e-f$ would be a good factor. Let $C, D$ be distinct components of $F^{\prime}-x$, and let $Y:=N_{F^{\prime}}(x) \cap V(C)$ and $Z:=N_{F^{\prime}}(x) \cap V(D)$. There is no edge in $G$ between a vertex of $Y$ and one of $Z$, for otherwise there were edges $e \in[x, y]_{F^{\prime}}$, $f \in[x, z]_{F^{\prime}}, h \in[y, z]_{G} \backslash E\left(F^{\prime}\right)$ for some $y \in Y, z \in Z$, and $\left(F^{\prime}-e-f\right)+h$ would be a good factor, a contradiction. In particular, $C$ and $D$ are the only components of $F^{\prime}-x$. Since $G$ is claw-free, $Y$ and $Z$ are complete in $G$. Without loss of generality, we may assume that there are at least three edges between $x$ and vertices of $Y$ (otherwise we swap the roles of $Y$ and $Z$ ). Then $Y$ must be complete in $F^{\prime}$ as well, for otherwise there would be edges $e \in[x, y]_{F^{\prime}}, f \in[x, z]_{F^{\prime}}, h \in[y, z]_{G} \backslash E\left(F^{\prime}\right)$, and so $\left(F^{\prime}-e-f\right)+h$ would be a good factor, a contradiction. It follows that there cannot be a pair $e, f$ of distinct edges between $x$ and $y \in Y$, for otherwise $F^{\prime}-e-f$ would be a good factor, a contradiction. So $|Y| \geq 3$. But then $F^{\prime}-[x, y]-[x, z]-e$ is a good factor for arbitrary $e \in[y, z]_{F^{\prime}} \neq \emptyset, y, z \in Y$, our final contradiction.

Lemma 9 deals with the existence of a connected even factor in 4-connected line graphs of multigraphs.

\section{Lemma 9}

Every 4-connected line graph of a multigraph contains a connected factor which has degree two or four at each vertex.

Proof Let $G$ be a multigraph such that $L(G)$ is 4-connected. Suppose that $x$ is a vertex of degree 3 in $G$. If a neighbor $y$ of $x$ has degree less than 3 , then $G-\{x, y\}$ must be edgeless, since $L(G)$ is 4-connected. In this case, the assertion of the theorem can be checked easily by exhaustion. So doubling an edge $e$ incident with $x$, i.e. adding a further, new edge $e^{+}$with the same endvertices as $e$, will not produce a vertex of degree less than four at one of its ends. So there exists a set $E^{\prime} \subseteq E(G)$ such that doubling each edge of $E^{\prime}$ (once) produces a graph $G^{\prime}$ without vertices of degree 3 , with $E\left(G^{\prime}\right)=E(G) \cup\left\{e^{+} \mid e \in E^{\prime}\right\}$, and with $V\left(G^{\prime}\right)=V(G)$. Furthermore, no edge $e \in E^{\prime}$ has endvertices of degree one or two in $G$. 
By [7], there exists a dominating circuit of $G$ which contains all vertices of degree at least 4 in $G^{\prime}$, and here we can achieve that if it contains exactly one of $e$ and $e^{+}$, then it contains $e$. Among all dominating circuits with these properties we choose one, say $F$, with as few edges as possible. It follows that if $F$ contains both edges $e$ and $e^{+}$for some $e \in E^{\prime}$, then $F-e-e^{+}$is disconnected. The edges of $F$ induce a dominating circuit $T$, which we orient according to one way of traversing the circuit, starting at an arbitrary vertex. Since $F-e-e^{+}$ is disconnected whenever $e$ and $e^{+}$are in $F$ for some $e \in E^{\prime}, e$ and $e^{+}$are oriented oppositely (if they are both in $F$ ).

Now we produce a sequence $T^{\prime}$ of edges of $G$ by inserting some of the edges not in $E(F)$ (not necessarily once) at some position into the sequence of edges corresponding to $T$, according to the following rules:

1) If $e$ and $f$ with $f=e^{+}$or $e=f^{+}$are consecutive on $T$, then we insert two edges of $E(G) \backslash E(F)$ incident with the outvertex of $e$ (i.e. the invertex of $f$ ) at the position in between $e$ and $f$ (such edges exist).

2) If $e$ and $f$, and $f^{+}$and $e^{+}$are both consecutive on $T$, then we insert an edge incident with the outvertex of $f^{+}$at the position in between $f^{+}$and $e^{+}$(such an edge exists).

The sequence $T^{\prime}$ need not be a circuit. Note that every inserted edge occurs at most twice in $T^{\prime}$ and all others occur once in $T^{\prime}$; those which have been inserted twice never occur consecutively in $T^{\prime}$. Neither $e$ and $e^{+}$nor $e^{+}$and $e$ are consecutive in $T^{\prime}$, and if $e$ and $f$ are consecutive in $T^{\prime}$, then $f^{+}$and $e^{+}$are not.

Now we construct $T^{\prime \prime}$ from $T^{\prime}$ by inserting sequentially the remaining edges: If there is an edge $e$ in $E(G)$ not inserted so far into $T$, then we insert it at a position between $f$ and $g$, whenever $e, f$ and $g$ have a common endvertex. If this is not possible, then $e$ has a common endvertex with the first and the last edge of $T^{\prime \prime}$, and we add $e$ at the end of $T^{\prime \prime}$. All edges inserted in the latter way into $T^{\prime}$ occur only once.

Finally, we construct $T^{\prime \prime \prime}$ from $T^{\prime \prime}$ by replacing each doubled edge $e^{+}, e \in E^{\prime}$, by the original edge $e$.

$T^{\prime \prime \prime}$ is a sequence of edges of $G$ with the following properties:

1) Any two consecutive edges have a common vertex, and the first and the last one have a common vertex.

2) Two consecutive edges of $T^{\prime \prime \prime}$ are distinct.

3) If $e, f \in E^{\prime}$ are consecutive in $T^{\prime \prime \prime}$, then $f$ and $e$ are not.

4) Every edge of $G$ occurs in $T^{\prime}$ at least once, at most $3 \cdot\left|E^{\prime}\right|$ edges occur twice, and no edge of $G$ occurs more than twice.

Therefore, the edges of $T^{\prime \prime \prime}$ form a connected factor of $L(G)$ with vertices of degree 2 or 4 , and with at most $3\left|E^{\prime}\right|$ vertices of degree 4 .

In general, one cannot expect an upper bound for $\left|E^{\prime}\right|$ better than the number $v_{3}(G)$ of vertices of degree 3 in $G$, which leads, according to the proof of Lemma 9, to an upper bound of $3 \cdot v_{3}(G)$ for the number of vertices of degree 4 in the factor. Unfortunately, this bound may equal $|V(L(G))|$, for example if $G$ is an essentially 4-edge-connected bipartite graph where one color class consists of vertices of degree 3 .

If one provides more structure on $G$, then one can improve this bound. For example, if in 
$G$ the vertices of degree 3 are independent, then one gets $\left|E^{\prime}\right| \leq v_{3}(G)$ by similar arguments as above. This implies, for example, that a 4-connected line graph with minimum degree 5 contains a connected factor with more than $2 / 3$ of its vertices having degree 2 and all others having degree 4 .

Now we are able to establish the main result of this section.

\section{Theorem 10}

Every 4-connected claw-free graph contains a connected factor which has degree two or four at each vertex.

Proof Let $G$ be a 4-connected claw-free graph. Then $\operatorname{cl}(G)$ is a 4-connected line graph. By Lemma $9, \operatorname{cl}(G)$ contains a connected factor which has degree two or four at each vertex. By Lemma $7, G$ contains a connected factor which has even degree at each vertex. Finally, by Lemma 8, the assertion follows.

By the results of [7] it is also possible to prove the stronger result that between every pair of distinct vertices in a 4-connected line graph there exists a spanning trail which passes every vertex at most twice.

\section{Factors consisting of a bounded number of paths}

In this section we prove that Conjecture 1 and 2 are equivalent to seemingly weaker conjectures in which the conclusion that $G$ is hamiltonian is replaced by the conclusion that $G$ contains a factor consisting of a number of paths bounded by a constant, or, more generally, by a function which is sublinear in the number of vertices of the graph. In particular we show that every $k$-connected claw-free graph is hamiltonian if and only if every $k$-connected claw-free graph is traceable, i.e. contains a Hamilton path. For convenience we use the term r-pathfactor for a factor consisting of at most $r$ paths. A path-factor is an $r$-path factor for some $r$, and its endvertices are the vertices of degree less than 2 of its components.

We start with an auxiliary result. Here a $k$-clique of a graph $G$ is a subset of $k$ vertices of $G$ inducing a complete subgraph in $G$.

\section{Lemma 11}

Let $k \geq 2$ be an integer. If there exists a $k$-connected nonhamiltonian claw-free graph on $n$ vertices, then there exists a $k$-connected nonhamiltonian claw-free graph on at most $2 n-2$ vertices containing a $k$-clique.

Proof Let $G$ be a $k$-connected nonhamiltonian claw-free graph on $n$ vertices, and assume that $G=\operatorname{cl}(G)$. Hence $G$ is the line graph of some triangle-free graph $H$. We may assume $k \geq 4$, since for If $k \leq 3$ the claw-freeness clearly implies that there is a $k$-clique in $G$. If all vertices of $H$ have degree at least 4 , then it is easy to see that $H$ is 4-edge connected; by the result of [14] $G$ is hamiltonian. If there is a vertex in $H$ with precisely one neighbor $u$, then the edges incident with $u$ induce a clique in $G$ with at least $k$ vertices. Hence we may assume there is a vertex $x$ of degree 2 or 3 in $H$. Therefore $G$ contains a vertex whose neighborhood 
consists of disjoint cliques $R$ and $Q$ with $|R| \geq|Q| \in\{1,2\}$. If some vertex of $G$ is contained in a $k$-clique, then we are done. Hence we may assume that $|R|=k-2$ and $|Q|=2$. Now consider two copies $G_{1}$ and $G_{2}$ of $G$ with the same fixed vertex $x$ called $x_{i}$ in $G_{i}(i=1,2)$ and the same partition of $N(x)$ into two cliques $Q_{i}, R_{i}$ in $G_{i}$ with $\left|Q_{i}\right|=2$ and $\left|R_{i}\right|=k-2$ for $i=1,2$, respectively. Define the graph $G^{\prime}$ on $2 n-2$ vertices obtained from $G_{1}$ and $G_{2}$ by deleting $x_{1}$ and $x_{2}$, and joining all vertices of $Q_{1}$ to all vertices of $Q_{2}$, and joining all vertices of $R_{1}$ to all vertices of $R_{2}$. Denote by $E^{\prime}$ the set of edges joining vertices of $G_{1}-x_{1}$ and $G_{2}-x_{2}$. Then one easily checks that $G^{\prime}$ is claw-free and $k$-connected, and that $G^{\prime}$ contains a $k$-clique. We complete the proof by showing that $G^{\prime}$ is nonhamiltonian.

Suppose to the contrary that $G^{\prime}$ has a Hamilton cycle $C$. Then $F_{i}:=C \cap\left(G_{i}-x_{i}\right)$ is a path-factor of $G_{i}-x_{i}$ with all endvertices in $Q_{i} \cup R_{i}$. Either $F_{1}$ contains no path between the vertices of $Q_{1}$, or $F_{2}$ contains no path between the endvertices, for otherwise these two paths, together with two edges of $E^{\prime}$, would form a proper subcycle of $C$, which is absurd. Without loss of generality, $F_{1}$ contains no path between the endvertices of $Q_{1}$.

Case 1. $Q_{1}$ contains no endvertex of $F_{1}$. Then $F_{1} \cup\left\{x_{1}\right\}$ is a path-factor of $G_{1}$ with all endvertices in the clique $R_{1} \cup\left\{x_{1}\right\}$.

Case 2. $Q_{1}$ contains endvertices of exactly one component $P$ of $F_{1}$. Then $Q_{1}$ contains precisely one endvertex of $P$, and hence $\left(F_{1}-P\right) \cup\left(P+x_{1}\right)$ is a path-factor of $G_{1}$ with all endvertices in the clique $R_{1} \cup\left\{x_{1}\right\}$.

Case 3. $Q_{1}$ contains endvertices of two distinct components $P \neq P^{\prime}$ of $F_{1}$. Then $\left(F_{1}-\right.$ $\left.P-P^{\prime}\right) \cup\left(P+x_{1}+P^{\prime}\right)$ is a path-factor of $G_{1}$ with all endvertices in the clique $R_{1}$.

Since a graph on at least 3 vertices is hamiltonian if and only if it has a path-factor with all endvertices being contained in the same clique, it follows in either case that $G_{1}$ is hamiltonian, a contradiction.

We use the above lemma to prove the following result.

\section{Theorem 12}

Let $k \geq 2$ and $r \geq 1$ be two integers. Then the following statements are equivalent.

(1) There is a $k$-connected claw-free nonhamiltonian graph.

(2) There is a $k$-connected claw-free graph without an $r$-path-factor.

Moreover, if there is an example for (1) on $n$ vertices, then there is an example for (2) with at most $(2 r+1)(2 n-2)$ vertices.

Proof It is clear that we only have to show that the existence of a $k$-connected claw-free nonhamiltonian graph on $n$ vertices implies the existence of a $k$-connected claw-free graph without an $r$-path-factor on at most $(2 r+1)(2 n-2)$ vertices.

Let $G$ be a $k$-connected claw-free nonhamiltonian graph on $n$ vertices. Then by Lemma 11 there is a $k$-connected claw-free nonhamiltonian graph $H$ on at most $2 n-2$ vertices containing a $k$-clique $Q$. We may assume that $H=\operatorname{cl}(H)$. Let $G_{r}$ be the graph obtained from $2 r+1$ disjoint copies of $H$ by joining all vertices corresponding to the $k$-clique $Q$ in all copies, forming a $(2 r+1) k$-clique. Clearly, $G_{r}$ is claw-free and $k$-connected and has at most $(2 r+1)(2 n-2)$ 
vertices. We complete the proof by showing that $G_{r}$ admits no $r$-path-factor. Suppose to the contrary that $P$ is an $r$-path-factor of $G_{r}$. Then $P$ has at most $2 r$ vertices of degree zero or one. Since $G_{r}$ contains $2 r+1$ disjoint copies of $H$, this implies that for at least one copy of $H, V(H) \backslash Q$ contains no endvertices of $P$. It is obvious that we can construct a Hamilton cycle in this copy of $H$, contradicting the assumption that $H$ is nonhamiltonian.

Theorem 12 has a number of interesting consequences, the first of which is obvious and given without proof.

\section{Corollary 13}

Let $k \geq 2$ be an integer. Then the following statements are equivalent.

(1) Every $k$-connected claw-free graph is hamiltonian.

(2) Every $k$-connected claw-free graph is traceable.

In particular Corollary 13 shows that Conjecture 2 is equivalent to the conjecture that every 4-connected claw-free graph is traceable. We can weaken the conclusion a little further. The next consequences of Theorem 12 can be obtained by examining the order of the graph $G_{r}$ in the proof of the theorem.

\section{Corollary 14}

Let $k \geq 2$ be an integer, and let $f(n)$ be a function of $n$ with the property that $\lim _{n \rightarrow \infty} \frac{f(n)}{n}=$ 0 . Then the following statements are equivalent.

(1) Every $k$-connected claw-free graph is hamiltonian.

(2) Every $k$-connected claw-free graph on $n$ vertices has an $f(n)$-path-factor.

(3) Every $k$-connected claw-free graph on $n$ vertices has a 2-factor with at most $f(n)$ components.

(4) Every $k$-connected claw-free graph on $n$ vertices has a spanning tree with at most $f(n)$ vertices of degree one.

(5) Every $k$-connected claw-free graph on $n$ vertices has a path of length at least $n-f(n)$.

Proof We only prove that (2) implies (1). The other cases are similar and left to the reader.

Suppose (2) is true and suppose there exists a $k$-connected claw-free nonhamiltonian graph on $m$ vertices. Then by Theorem 12 there is a $k$-connected claw-free graph $G_{r}$ without an $r$-path-factor on $n_{r} \leq(2 r+1)(2 m-2)$ vertices. If we let $r$ tend to infinity, then $G_{r}$ is a graph on $n_{r}$ vertices without an $r$-path-factor, while $\lim _{r \rightarrow \infty} \frac{r}{n_{r}} \geq \frac{1}{4 m-4}$ for a fixed integer $m>1$. This contradicts the assumption that (2) is true. 
In particular Corollary 14 shows that Conjecture 2 is true if one could show that, e.g., every 4-connected claw-free graph on $n$ vertices admits a factor consisting of a number of paths which is sublinear in $n$.

Recently, in [4] it has been shown that a claw-free graph $G$ has an $r$-path-factor if and only if $\operatorname{cl}(G)$ has an $r$-path-factor. Similarly, in [12] it has been shown that a claw-free graph $G$ has a 2-factor with at most $r$ components if and only if $\operatorname{cl}(G)$ has such a 2-factor. These results immediately imply the equivalence of the following statements related to Conjecture 1.

\section{Corollary 15}

Let $k \geq 2$ be an integer, and let $f(n)$ be a function of $n$ with the property that $\lim _{n \rightarrow \infty} \frac{f(n)}{n}=$ 0 . Then the following statements are equivalent.

(1) Every k-connected line graph is hamiltonian.

(2) Every $k$-connected line graph on $n$ vertices has an $f(n)$-path-factor.

(3) Every k-connected line graph on $n$ vertices has a 2-factor with at most $f(n)$ components.

In particular Corollary 15 shows that Conjecture 1 is true if one could show that, e.g., every 4-connected line graph on $n$ vertices admits a 2-factor consisting of a number of components which is sublinear in $n$. The equivalences between (1) and (2) of Corollary 14 and of Corollary 15 appear also in a sequence of equivalences in [9].

\section{References}

[1] J. A. Bondy and U.S. R. Murty, Graph Theory with Applications. Macmillan, London and Elsevier, New York (1976).

[2] J. Brousek, Z. Ryjáček and I. Schiermeyer, Forbidden subgraphs, stability and hamiltonicity. Preprint (1997).

[3] F. Harary and C. St. J. A. Nash-Williams, On eulerian and hamiltonian graphs and line graphs. Canad. Math. Bull. 8 (1965) 701-710.

[4] S. Ishizuka, Closure, path factors and path coverings in claw-free graphs. Preprint (1998).

[5] B. Jackson, Hamilton cycles in 7-connected line graphs. Preprint (1989).

[6] B. Jackson and N. C. Wormald, $k$-Walks of graphs. Australasian Journal of Combinatorics 2 (1990) 135-146.

[7] M. Kriesell, All 4-connected line graphs of claw-free graphs are hamiltonian-connected. Preprint (1998).

[8] S. Kundu, Bounds on the number of disjoint spanning trees. J. Combin. Theory B 17 (1974) 199-203.

[9] M. Kochol, Sublinear defect principle in graph theory. Manuscript (1999).

[10] M. M. Matthews and D. P. Sumner, Hamiltonian results in $K_{1,3}$-free graphs. J. Graph Theory 8 (1984) 139-146. 
[11] Z. Ryjáček, On a closure concept in claw-free graphs. J. Combin. Theory B 70 (1997) 217-224.

[12] Z. Ryjáček, A. Saito and R. H. Schelp, Closure, 2-factors and cycle coverings in claw-free graphs. J. Graph Theory, To appear.

[13] C. Thomassen, Reflections on graph theory. J. Graph Theory 10 (1986) 309-324.

[14] S. Zhan, Hamiltonian connectedness of line graphs. Ars Combinatoria 22 (1986) 89-95.

[15] S. Zhan, On hamiltonian line graphs and connectivity. Discrete Math. 89 (1991) 89-95. 